\title{
Reduction of phase jitter in dispersion-managed systems by in-line filtering
}

\author{
David Boivin, Marc Hanna, and Pierre-Ambroise Lacourt \\ GTL-CNRS Telecom, Unité Mixte de Recherche du Centre National de la Recherche Scientifique 6603, \\ 2-3 Rue Marconi, 57070 Metz, France
}

Jean-Pierre Goedgebuer

Laboratoire d'Optique P. M. Duffieux, Unité Mixte de Recherche du Centre National de la Recherche Scientifique 6603, Route de Gray, 25030 Besançon Cedex, France

\begin{abstract}
The influence of in-line filters on the phase jitter of chirped optical pulses propagating in arbitrary dispersionmanaged systems is studied with a semianalytic moment method. Because of its stabilizing effect on the amplitude, filtering reduces the nonlinear phase jitter that accumulates through self-phase modulation. As in the case of constant-dispersion soliton links, we observe that the phase variance grows only linearly with distance in the presence of filtering. Phase jitter reduction is observed and accurately predicted by the moment method in two dispersion-managed systems with different levels of nonlinearity and filter strength.
\end{abstract}

Variations on the differential phase-shift-keying (DPSK) modulation format have recently allowed the demonstration of impressive transmission capacities and represent a promising technique for further improving the performance of optical communication systems. ${ }^{1}$ In these formats data are encoded in an optical phase difference between adjacent bits. It has been shown that this type of modulation offers several advantages, namely, the reduction of the penalty caused by nonlinear effects, ${ }^{2}$ a good tolerance to narrowband filtering, a high spectral efficiency in the quadrature DPSK implementation, ${ }^{3}$ and the possibility of using balanced detection to enhance the sensitivity of the receiver. In these systems the error-free transmission distance is limited by random fluctuations of the phase. Physically, phase jitter arises from amplified spontaneous emission noise that is added to the signal at each amplification stage along the link. Amplitude-to-phase noise conversion occurs through the self-phase modulation (SPM) effect, producing a phase variance that grows as the cube of the propagation distance.

Phase jitter has been studied in several types of communication system by use of the soliton perturbation theory, ${ }^{4}$ the variational approach, ${ }^{5}$ and the moment method. ${ }^{6,7}$ It was numerically shown that synchronous modulation does not reduce phase jitter efficiently. ${ }^{8}$ In-line filtering was first proposed to reduce the timing jitter $^{9}$ and was experimentally demonstrated in a dispersion-managed (DM) system. ${ }^{10}$ It was later shown to stabilize the phase as well in the case of constant-dispersion soliton links. ${ }^{4}$ The physical mechanism for phase control is as follows: Optical filters stabilize the pulse spectral width, and hence peak power, since these variables are coupled by nonlinear soliton propagation. The control of the peak power then acts on the stability of the phase accumulated by SPM. In DM systems the periodic chirping of the pulse complicates the analysis, ${ }^{11}$ and the effect of filtering on the phase jitter must be reconsidered. In this Letter a semianalytic moment method is used to study this phenomenon in single-channel DM links, with the only assumptions being that the frequency chirp remains linear and the filters are Gaussian shaped. This analysis takes into account only the contribution of amplified spontaneous emission noise and SPM, which appears to be the dominant source of phase jitter even in wavelength-division multiplexing systems. ${ }^{5}$ Our method, which requires a single numerical resolution of the propagation equation, is validated by comparison with Monte Carlo simulations. We show that filtering still allows the reduction of phase noise in two examples of DM systems.

The propagation of a single optical pulse in a filtered amplified link is described by the nonlinear Schrödinger equation:

$$
\begin{aligned}
i \frac{\partial u}{\partial z}-\frac{1}{2}\left[\beta_{2}(z)-i b(z)\right] \frac{\partial^{2} u}{\partial t^{2}} & +\gamma(z)|u|^{2} u \\
& =i g(z) u+\hat{F}(z, t),
\end{aligned}
$$

where $u(z, t)$ is the pulse envelope, $g(z)$ is the local net gain, $\beta_{2}(z)$ is the local group-velocity dispersion, $\gamma(z)$ is the local nonlinearity, and $b(z)$ is the filtering factor. The noise source term is described by its autocorrelation function:

$$
\left\langle\hat{F}(z, t) \hat{F}^{*}\left(z^{\prime}, t^{\prime}\right)\right\rangle=2 g_{0} \hbar \omega_{0} n_{\mathrm{sp}}(z) \delta\left(z-z^{\prime}\right) \delta\left(t-t^{\prime}\right),
$$

where $n_{\mathrm{sp}}$ is the spontaneous emission factor, $g_{0}$ is the amplification coefficient inside the amplifier, and $\hbar \omega_{0}$ is the photon energy at the signal frequency. Using the same definitions for power $P$, energy $E$, and phase $\Phi$ as in Ref. 7 and the same assumption of a 
pulse exhibiting mostly linear chirp with $\arg (u)(z, t) \approx$ $\phi_{0}(z)+\phi_{2}(z)\left(t-t_{0}\right)^{2}$, we obtain the following dynamic equations:

$$
\begin{aligned}
& \frac{\mathrm{d} \Phi}{\mathrm{d} z}=-\beta_{2} \phi_{2} \Phi+\gamma P \\
&+\frac{i}{E} \int_{-\infty}^{+\infty}[\arg (u)-\Phi]\left(u \hat{F}^{*}-u^{*} \hat{F}\right) \mathrm{d} t \\
&-\frac{1}{2 E} \int_{-\infty}^{+\infty} u^{*} \hat{F}+u \hat{F}^{*} \mathrm{~d} t, \\
& \frac{\mathrm{d} P}{\mathrm{~d} z}=2\left[g+\beta_{2} \phi_{2}-\frac{b(z)}{2 E} \int_{-\infty}^{+\infty}\left|u_{t}\right|^{2} \mathrm{~d} t\right] P \\
&+\frac{i}{E} \int_{-\infty}^{+\infty}\left(2|u|^{2}-P\right)\left(u \hat{F}^{*}-u^{*} \hat{F}\right) \mathrm{d} t,
\end{aligned}
$$

where only first-order terms in $b$ were kept. The power is given by

$$
\begin{aligned}
P= & \left\{P_{0}+i \int_{0}^{z}\left[\frac{1}{E A_{1}} \int_{-\infty}^{+\infty}\left(2|u|^{2}-P\right)\right.\right. \\
& \left.\left.\times\left(u \hat{F}^{*}-u^{*} \hat{F}\right) \mathrm{d} t\right] \mathrm{~d} z_{1}\right\} A_{1},
\end{aligned}
$$

where

$$
A_{1}(z)=\exp \left\{\int_{0}^{z} 2\left[g+\beta_{2} \phi_{2}-\frac{b(z)}{2 E} \int_{-\infty}^{+\infty}\left|u_{t}\right|^{2} \mathrm{~d} t\right] \mathrm{d} z_{1}\right\} .
$$

The filtering term changes the overall behavior of the solution. The correction acts on the multiplicative term $A_{1}(z)$, dampening the fluctuations of $P$. It results in a smaller power noise, and hence nonlinear phase noise, since it is induced by power fluctuations through SPM. The remaining derivation of the phase variance yields $\sigma_{\Phi}^{2}=\left\langle\Phi_{1}^{2}\right\rangle+\left\langle\Phi_{2}^{2}\right\rangle+\left\langle\Phi_{3}^{2}\right\rangle+2\left\langle\Phi_{1} \Phi_{2}\right\rangle$, where $\Phi_{1}$ is the nonlinear contribution and $\Phi_{2}$ and $\Phi_{3}$ are the linear contributions. This derivation is described in Ref. 7 and yields the same result with the exception of the modified $A_{1}(z)$ term. The obtained expression for the phase jitter depends on only the link parameters and the field unperturbed by noise.

To demonstrate the versatility of the moment method, we apply it to two filtered DM systems with different levels of nonlinearity and filter strength and check our model by comparing it with direct Monte Carlo simulations. The propagation of a single pulse in a noisy channel is simulated 512 times to compute the phase variance. The optical links are formed by alternating spans of anomalous-dispersion fiber 1 and normal-dispersion fiber 2 . The DM soliton link operates at a high peak power and an average dispersion of $D_{a}=0.5 \mathrm{ps} / \mathrm{nm} \mathrm{km}$, whereas the quasi-linear system operates at low power with zero average dispersion. Unchirped pulses are launched at the midpoint of the normal-dispersion span. The system's parameters are summarized in Table 1. For both systems, optical amplifiers followed by Gaussian filters are placed every $z_{a}=60 \mathrm{~km}$ along the link, with $n_{\mathrm{sp}}=1.5$. The FWHM bandwidth $B$ of the filters in terms of optical power is related to the filtering factor by the relation $b=\ln (2) /\left(\pi^{2} B^{2} z_{a}\right)$. Because filters remove some signal power in the wings of the pulse spectra, the gain of the amplifiers must be increased slightly to conserve energy.

The standard deviation of the phase in the DM soliton system is plotted in Fig. 1 for different filter bandwidths. The $500-\mathrm{GHz}$ filter case corresponds to an almost unfiltered system since the signal bandwidth is $\sim 15 \mathrm{GHz}$. We observe that the phase standard deviation is greatly reduced by narrowband filtering. It exhibits a $z^{1 / 2}$ dependence in the case of $45-\mathrm{GHz}$ filters, which is similar to the constant dispersion soliton case. ${ }^{4}$ Although the moment method does not accurately predict the locations of the slight oscillations, there is satisfactory agreement with Monte Carlo simulations. The mismatch in oscillation locations might be due to a small amount of nonlinear chirping in the pulse and higher-order terms in the filtering factor $b$. The filtering is not as efficient in suppressing phase jitter in the case of the quasi-linear system, as shown in Fig. 2. The explanation for this is that the system is operating at low power with a weak nonlinear phase noise contribution. Since the filters essentially act by stabilizing the power of the pulses, therefore reducing the nonlinear part of phase noise

Table 1. Numerical Values of the System Parameters

\begin{tabular}{lcc} 
& DM Soliton DM Quasi-Linear \\
\hline Pulse shape & $\mathrm{sech}^{2}$ & Gaussian \\
FWHM pulse width (ps) & 20 & 20 \\
Pulse peak power (mW) & 7.2 & 0.5 \\
Fiber 1 dispersion & & \\
$\quad$ (ps /nm km) & 11 & 16 \\
Fiber 1 length (km) & 30 & 50 \\
Fiber 1 effective area $\left(\mu \mathrm{m}^{2}\right)$ & 50 & 80 \\
Fiber 2 dispersion & & \\
$\quad$ (ps/nm km) & -10 & -80 \\
Fiber 2 length (km) & 30 & 10 \\
Fiber 2 effective area $\left(\mu \mathrm{m}^{2}\right)$ & 50 & 45 \\
\hline
\end{tabular}

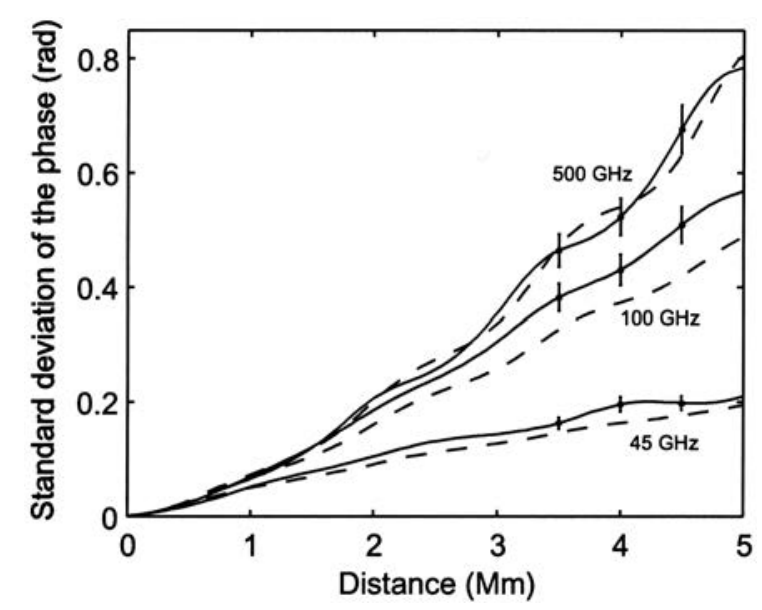

Fig. 1. Standard deviation of the phase as a function of distance for the DM soliton system with optical filter bandwidths of 500, 100, and $45 \mathrm{GHz}$. Solid curves, Monte Carlo simulation results; dashed curves, moment method results. 


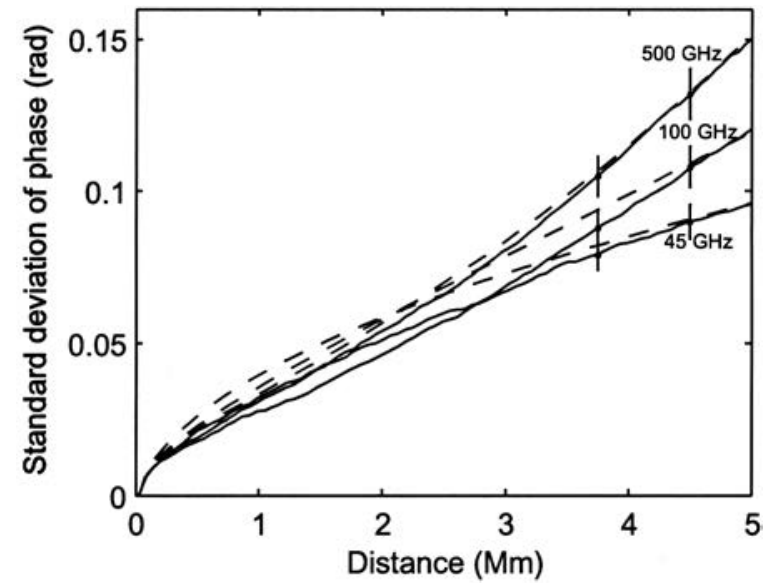

Fig. 2. Standard deviation of the phase as a function of distance for the DM quasi-linear system with optical filter bandwidths of 500, 100, and $45 \mathrm{GHz}$. Solid curves, Monte Carlo simulation results; dashed curves, moment method results.

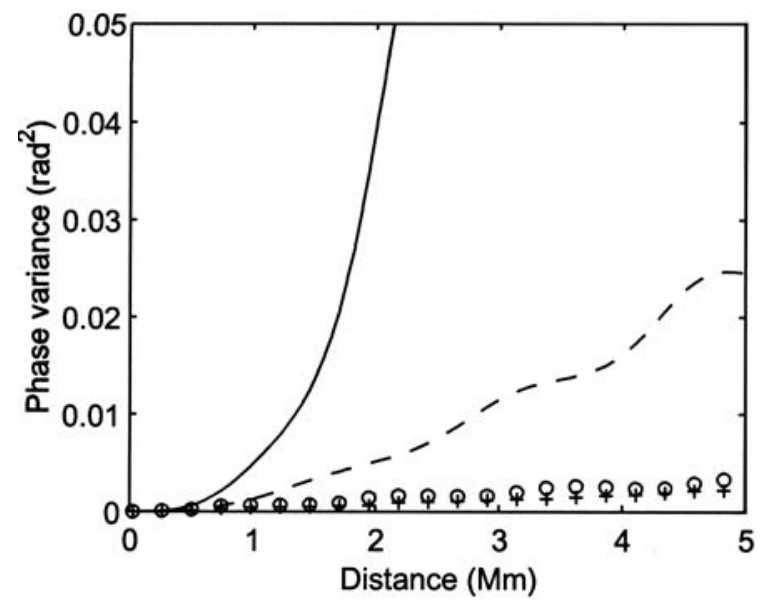

Fig. 3. Contribution of the nonlinear phase noise to the overall phase jitter for the DM soliton system. Solid curve, nonlinear phase variance with $500-\mathrm{GHz}$ filters; dashed curve, nonlinear phase variance with $45-\mathrm{GHz}$ filters; circles, linear phase variance for 500-GHZ filters; crosses, linear phase variance for $45-\mathrm{GHz}$ filters.

mediated by SPM, they do not have an important effect on the quasi-linear system.

To further clarify this stabilizing mechanism, we plotted in Fig. 3 the linear and nonlinear contributions to phase jitter in the case of the weakly and strongly filtered DM soliton system as given by the moment method. The linear contribution corresponds to setting $\gamma=0$ in Eq. (1). For 500-GHz filters the nonlinear contribution to the phase variance reaches $0.6 \mathrm{rad}^{2}$ at $5 \mathrm{Mm}$ and grows as $z^{3}$. When $45-\mathrm{GHz}$ filters are used, the nonlinear phase variance is reduced to $0.025 \mathrm{rad}^{2}$ at $5 \mathrm{Mm}$, growing approximately linearly with distance. The linear contribution to phase variance is almost unchanged under strong filtering.

Assuming a perfect phase receiver and a Gaussian probability density function, the standard deviation that yields a symbol error rate of $10^{-9}$ is $0.26 \mathrm{rad}$ for binary DPSK and $0.13 \mathrm{rad}$ for quadrature DPSK. For the DM soliton system, narrowband filtering allows error-free transmission over $5 \mathrm{Mm}$ for binary DPSK and over $2.5 \mathrm{Mm}$ for quadrature DPSK. For the quasi-linear system no filtering is required for binary DPSK, whereas $45-\mathrm{GHz}$ filters allow the error-free transmission of quadrature DPSK over $5 \mathrm{Mm}$.

We have shown that in-line filtering is an efficient way to reduce phase jitter in single-channel DM systems. A model based on the moment method was developed to evaluate phase jitter for arbitrary pulse shapes in such systems and is in good agreement with Monte Carlo simulations at a fraction of the computational cost, providing a useful tool for the performance assessment of DPSK systems. Since in-line filtering acts through the stabilization of amplitude, a reduction of the contribution of cross-phase modulation to phase jitter in multichannel systems ${ }^{5}$ is also expected.

D. Boivin's e-mail address is dboivin@georgiatechmetz.fr

\section{References}

1. T. Hoshida, O. Vassilieva, K. Yamada, S. Choudhary, R. Pecqueur, and H. Kuwahara, J. Lightwave Technol. 20, 1989 (2002).

2. J. Leibrich, C. Wree, and W. Rosenkranz, IEEE Photon. Technol. Lett. 14, 155 (2002).

3. H. Kim and R.-J. Essiambre, IEEE Photon. Technol. Lett. 15, 769 (2003).

4. M. Hanna, H. Porte, W. T. Rhodes, and J.-P. Goedgebuer, Opt. Lett. 24, 732 (1999).

5. C. J. McKinstrie, C. Xie, and C. Xu, Opt. Lett. 28, 604 (2003).

6. V. S. Grigoryan, C. R. Menyuk, and R. M. Mu, J. Lightwave Technol. 17, 1347 (1999).

7. M. Hanna, D. Boivin, P.-A. Lacourt, and J.-P. Goedgebuer, J. Opt. Soc. Am. B 21, 24 (2004).

8. O. Leclerc and E. Desurvire, Opt. Lett. 23, 1453 (1998).

9. A. Mecozzi, J. D. Moores, H. A. Haus, and Y. Lai, Opt. Lett. 16, 1841 (1991).

10. E. A. Golovchenko, J. M. Jacob, A. N. Pilipetskii, C. R. Menyuk, and G. M. Carter, Opt. Lett. 22, 289 (1997).

11. C. J. McKinstrie, J. Opt. Soc. Am. B 19, 1275 (2002). 